\title{
Do absorption and realistic distraction influence performance of component task surgical procedure?
}

\author{
Jon R. Pluyter · Sonja N. Buzink · Anne-F. Rutkowski • \\ Jack J. Jakimowicz
}

Received: 18 February 2009/Accepted: 10 August 2009/Published online: 30 September 2009

(c) The Author(s) 2009. This article is published with open access at Springerlink.com

\begin{abstract}
Background Surgeons perform complex tasks while exposed to multiple distracting sources that may increase stress in the operating room (e.g., music, conversation, and unadapted use of sophisticated technologies). This study aimed to examine whether such realistic social and technological distracting conditions may influence surgical performance.

Methods Twelve medical interns performed a laparoscopic cholecystectomy task with the Xitact LC 3.0 virtual reality simulator under distracting conditions (exposure to music, conversation, and nonoptimal handling of the laparoscope) versus nondistracting conditions (control condition) as part of a $2 \times 2$ within-subject experimental design. Results Under distracting conditions, the medical interns showed a significant decline in task performance (overall task score, task errors, and operating time) and significantly increased levels of irritation toward both the assistant handling the laparoscope in a nonoptimal way and the sources of social distraction. Furthermore, individual differences in cognitive style (i.e., cognitive absorption and
\end{abstract}

J. R. Pluyter $(\bowtie)$ A A.-F. Rutkowski

Department of Information Systems and Management,

Tilburg University, Warandelaan 2, 5037 AB Tilburg,

The Netherlands

e-mail: J.R.Pluyter@uvt.nl

S. N. Buzink - J. J. Jakimowicz

Department of Surgery, Catharina Hospital Eindhoven,

Michelangelolaan 2, P.O. Box 1350, 5602 ZA Eindhoven,

The Netherlands

S. N. Buzink · J. J. Jakimowicz

Faculty of Industrial Design Engineering,

Delft University of Technology, Landbergstraat 15 ,

2628 CE Delft, The Netherlands need for cognition) significantly influenced the levels of irritation experienced by the medical interns.

Conclusion The results suggest careful evaluation of the social and technological sources of distraction in the operation room to reduce irritation for the surgeon and provision of proper preclinical laparoscope navigation training to increase security for the patient.

Keywords Laparoscope navigation - Laparoscopy · Simulator - Social distraction - Technological distraction . Training

Minimally invasive surgery has turned the operating room into an even more complex environment involving multidimensional information technologies (IT) that put extraneous physical and cognitive demands on the medical staff $[1,2]$, cause new forms of error [3], and accordingly may increase stress for the medical staff.

Music, external staff, phone calls, and case-irrelevant communication are known sources of social distraction in the operating room that may affect concentration and performance for the untrained surgeon [4-7]. Although generally assumed to be so, proficiency in tissue manipulation does not necessarily imply skillfulness in laparoscope navigation, a task often performed by the least experienced member of the surgical team [8]. Such technological distraction, to say nothing of interruption, requires the surgeon to refocus his or her attention repetitively, causing extra mental strain. Congruently, Healey et al. [5] observed that poor image focus "distracted and interrupted surgical teams from their work." In the current operating room setting, dense IT usage is an additional potential source of distraction and error that to date remains 
rather unexplored. We refer to such technological distractions as "distraction brought by IT."

Previous studies $[9,10]$ have posed arithmetic problems as a primary distracting source, affecting the span of the short-term memory, and thus demonstrating significant adverse effects on surgical performance. Realistic distractions, however, probably have a different impact on surgical performance because they are more likely to be blocked out.

This report describes the consequences of distracting sounds and nonoptimal laparoscope navigation in a lab experiment that represent realistic social and technological distractions present in the operating room. We hypothesize that under distracting conditions, objective surgical performance will be lower (H1) and, inversely, that irritation and stress levels will be higher (H2) than under nondistracting conditions.

Additionally, distraction has a different impact on individuals as a function of their cognitive styles [11]. Cognitive styles such as cognitive absorption (CA) and need for cognition (NFC) have been used to assess individual differences in a technology usage context. Agarwal and Karahanna [12] define cognitive absorption as "a state of deep involvement with software" in which episodes of total attention may lead to "imperviousness to normally distracting events," among other responses [12].

In our study, the CA scale was adapted, allowing IT-specific (i.e., surgical simulator-specific) measurement. The need for cognition represents "the tendency for an individual to engage in and enjoy thinking" [13] and appears to be positively related to CA [14]. Therefore, we hypothesize that participants with combined high scores on NFC and IT-specific CA will show higher objective performance and lower levels of irritation and stress when not distracted (H3a, H4a) and when distracted (H3b, H4b) than participants with low scores on the same cognitive styles constructs.

\section{Materials and methods}

In this study, 12 medical trainees took part in a 2 (proper vs nonoptimal laparoscope navigation) $\times 2$ (social distraction of music and conversation vs no social distraction) according to a within-subject experimental design. All the participants were selected on the basis that they had no simulator or clinical experience performing laparoscopic procedures, thus forming a baseline. They participated on a voluntary basis. Considering the expected learning curve of the participants [15], testing was limited to the two most extreme experimental conditions, namely, the socialtechnological distracting (D) condition (nonoptimal laparoscope navigation and social distraction) and the nondistracting (ND) condition (proper laparoscope navigation and no social distraction).

\section{Simulator}

The Clip and Cut $(\mathrm{C} \& \mathrm{C})$ module of the Xitact LC 3.0 virtual reality simulator (Xitact SA, Morges, Switzerland) was used. The $\mathrm{C} \& \mathrm{C}$ module consists of five exercises. The final exercise, the clip and cut assessed (CCA) task, covers the entire clip and cut procedure in a nontutored version, thus being closest to reality. Task completion, task errors, economy of movement, and time required to complete the procedure were recorded by the simulator.

\section{Blood pressure device}

Blood pressure (BP) and heart rate (HR) were used for stress measurement $[16,17]$. In this study the Stabil-OGraph (I.E.M. GmbH, Stolberg, Germany) was used to assess both factors. The Stabil-O-Graph is a noninvasive measuring device that was validated according to the electromagnetic compatibility (EC) directive and carries a conformite europeene (CE) mark.

\section{Distraction}

Under social-technological distracting conditions (Fig. 1: experimental condition [D]), the subjects were exposed to a standardized combination of music ( 2 popular songs) mixed in parallel with a $30 \mathrm{~s}$ of case-irrelevant communication. Together, the two conditions represented social distraction. To ensure that all the participants were fully exposed to the case-irrelevant communication, the conversation was placed within the first $70 \mathrm{~s}$ of the audio file. Additionally, the laparoscope was manipulated using a standardized protocol to provide a nonoptimal view (nonoptimal laparoscope navigation) by presentation of the cystic duct and cystic artery on the same horizontal level, representing technological distraction.

All the participants were assisted by the same assistant, an accomplice trained to act in a standardized and controlled way during the study. The sessions took place in a separate room to ensure that the participants were not distracted by other sources.

\section{Procedure}

The participants were told that they would perform a part of the laparoscopic cholecystectomy procedure on the simulator. Next, BP and HR were measured. The participants filled out the first part of the questionnaire (Fig. 1). Baseline BP and HR were reassessed to minimize the bias caused by case-irrelevant sources (e.g., hurrying toward the 


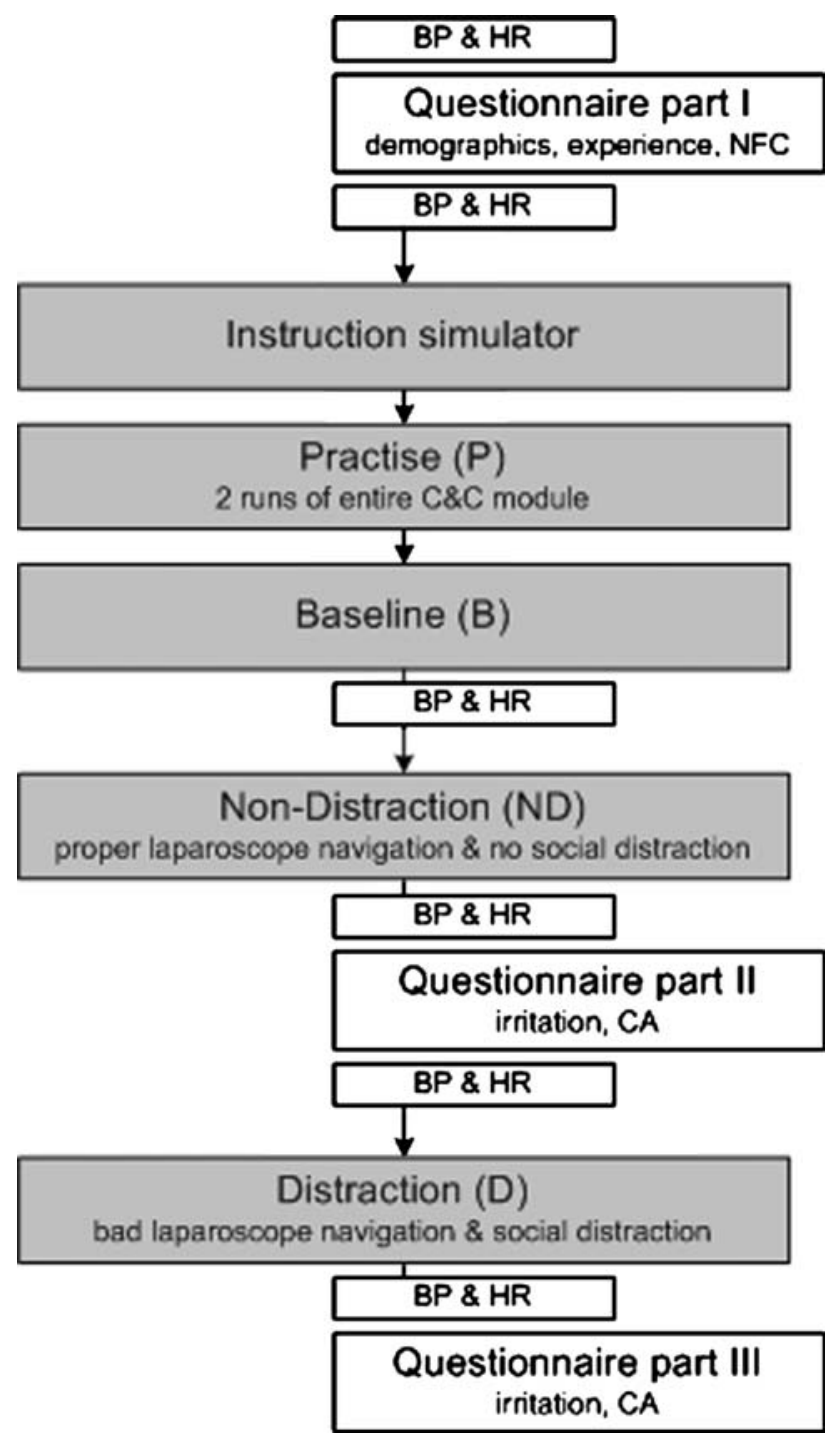

Fig. 1 Experimental procedure. All 12 participants performed in the same order, starting with practice $(\mathrm{P})$ and baseline performance (B), followed by the two experimental conditions, namely, nondistracting (ND) and distracting (D) conditions

simulator room). Subsequently, the participants received an introduction to the simulator and then performed two runs of all five C\&C module tasks (Fig. 1: practice [P]). During the first run, each task was explained using a demo video provided by the manufacturer accompanied by a standardized verbal instruction by the researcher.

To minimize the effects of unfamiliarity with the simulator or the task, only the results for the final task of the second run were used to assess the baseline performance level (Fig. 1: baseline [B]). Both BP and HR were measured to monitor the participants' physiologic state after baseline performance.

Next, the participants were instructed to perform the CCA task with the assistant handling the laparoscope. The assistant held the laparoscope without actually manipulating the view by using the fixed camera position of the simulator (Fig. 1: experimental condition [ND]). The co-action of the assistant holding the laparoscope was required to maintain the interaction effect between the performing participant and the laparoscope navigator [18].

Performance was assessed, and directly after completion of the task, HR and BP were measured. The participants then filled out the second part of the questionnaire. Again, HR and BP were measured to check whether both values had converged to their normal physiologic equilibrium. Subsequently, the participants were instructed to perform the CCA task again. The assistant manipulated the laparoscope view using the standardized protocol for nonoptimal laparoscope navigation representing technological distraction, and the participant was exposed to the social distraction during execution of the task (experimental condition [D]). Performance was assessed, and directly after completion of the task, BP and HR were measured. Finally, the participants filled out the third part of the questionnaire.

\section{Measures}

Besides the objective performance scores recorded by the simulator and the physiologic measurement of stress, the perceived irritation toward the simulator, the noise, and the assistant $[19,20]$ together with overall perceived irritation [19], NFC [13], and IT-specific CA [12] was measured via a survey using a 7-point Likert scale.

Statistical analysis

For the statistical analysis of the data, SPSS 15.0 for windows (SPSS Inc., Chicago, IL, USA) was used. The Wilcoxon signed ranks test was used to assess the intercondition (pairwise) comparison. The Mann-Whitney $U$ test was used to assess differences between groups based on their levels of NFC and IT-specific CA. The level of statistical significance was set at an alpha of 0.05 or less. A $p$ value of 0.05 to 0.07 indicated a significant tendency of the results.

\section{Results}

Comparison among conditions

Table 1 provides an overview of the objective performance measures across the different conditions. The baseline task score was significantly higher $(z=-1.926 ; p=0.05)$ than the practice score (Table 2 ), and the total time required was significantly lower $(z=-2.432 ; p=0.01)$ than during practice. This may indicate that a learning effect had occurred. 
Table 1 Objective task performance on the simulator: overview

\begin{tabular}{|c|c|c|c|c|c|c|c|c|}
\hline & \multicolumn{2}{|l|}{$\mathrm{P}$} & \multicolumn{2}{|l|}{ B } & \multicolumn{2}{|l|}{ ND } & \multicolumn{2}{|l|}{$\mathrm{D}$} \\
\hline & M & SD & M & SD & M & SD & M & $\mathrm{SD}$ \\
\hline Task score & 73.00 & 19.35 & 115.75 & 63.02 & 112.50 & 59.05 & 53.92 & 51.78 \\
\hline Task completion & 91.25 & 8.29 & 92.50 & 11.18 & 90.42 & 14.69 & 82.92 & 21.69 \\
\hline Task errors & 20.00 & 15.52 & 12.50 & 29.50 & 7.08 & 8.908 & 35.83 & 30.74 \\
\hline Total time (s) & 124.92 & 43.52 & 95.25 & 25.46 & 98.67 & 30.17 & 111.25 & 22.19 \\
\hline
\end{tabular}

$P$ practice, $B$ baseline performance, $N D$ nondistracting condition, $D$ distracting condition, $M$ mean, $S D$ standard deviation

Table 2 Objective performance: comparison among conditions

\begin{tabular}{llll}
\hline & P vs. B & B vs. D & ND vs. D \\
\hline Task score & 0.05 & ST & 0.05 \\
Task completion & NS & NS & NS \\
Task errors & NS & 0.02 & 0.03 \\
Total time (s) & 0.01 & ST & NS \\
\hline
\end{tabular}

$p$ Values are based on the Wilcoxon signed ranks test

$P$ practice, $B$ baseline performance, $N D$ nondistracting condition, $D$ distracting condition, $N S$ not significant, $S T$ significant tendency $(0.05<p \leq 0.07)$

No significant differences in objective performance could be reported between the baseline and the nondistracting conditions. It could be speculated that the expected learning effect was neutralized by the presence of the assistant [18]. The task error score was significantly higher under the social-technological distracting conditions $(z=-2.255 ; p=0.02)$ than under the baseline conditions (Table 2). Furthermore, there was a significant tendency toward a lower task score $(z=-1.867 ; p=0.06)$ and a longer total time $(z=-1.805 ; p=0.07)$ under the distracting conditions.

The task score was significantly lower $(z=-1.961$; $p=0.05)$ and the task error score significantly higher $(z=-2.162 ; p=0.03)$ under the distracting conditions than under the nondistracting conditions. Moreover, under the distracting conditions, the participants appeared significantly more irritated by the noise $(z=-2.820$; $p=0.005)$ and the assistant $(z=-2.263 ; p=0.02)$ than under the nondistracting conditions.

No significant differences could be reported for perceived irritation toward the simulator and overall perceived irritation. No significant differences among the different conditions could be observed for task completion or economy of movement. Analysis of the physiologic stress measures produced inconsistent results most likely biased toward case-irrelevant sources. Physiologic stress measurement thus seemed not to be applicable for measuring acute stress in the minimal paradigm of this study and therefore was excluded from further analysis.
Individual differences in cognitive styles

Categorizing based on IT-specific CA (medium: $n=6$ vs high: $n=4)$ measured after distracting conditions showed that medium cognitively absorbed participants (mean rank $[\mathrm{mrk}]=7.42$ ) were significantly more irritated by the noise under distracting conditions $(z=-2.467 ; p=0.01)$ than were the highly absorbed participants $(\mathrm{mrk}=2.63)$.

Categorizing based on the NFC (low: $n=7$ vs high: $n=5$ ) showed that the participants with a low NFC $(\mathrm{mrk}=8.14)$ reported a significantly higher score for irritation toward the simulator $(z=-1.898 ; p=0.05)$ than the participants with a high NFC $(\mathrm{mrk}=4.20)$ under nondistracting conditions. Furthermore, under nondistracting conditions, the participants with a low NFC $(\mathrm{mrk}=8.36)$ showed a significantly higher overall perceived level of irritation $(z=-2.115 ; p=0.03)$ than did the participants with a high NFC $($ mrk $=3.90)$. Similar results could be reported under distracting conditions $(\mathrm{mrk}=8.64$ for low NFC and 3.50 for high NFC; $z=-$ $2.44 ; p=0.01)$. All but hypothesis $3 \mathrm{a}$ and $\mathrm{b}$ could be verified.

\section{Discussion}

This study demonstrates the adverse effect of realistic social and technological distracting conditions on surgical performance. Overall, the results are in line with previous research using similar or different sources of distraction [9, 21]. Notably, highly absorbed participants indicated significantly less irritation toward the social distraction, which could be attributed to their higher level of imperviousness to normally distracting sources [12].

\section{Clinical implications}

The results of this study have three major implications for improving security in the operating room. First, the consequences of social distraction cannot be underestimated. Effort should be put into increasing the awareness among operating room staff regarding the impact of social 
distraction [4]. Accordingly, social distraction in the operating room should be considered carefully to avoid adverse events. This should include untangling the relationship between various (combinations of) possibly distracting sources and surgical performance. For example, music may have a positive impact under certain circumstances [22] but may have an adverse effect in combination with case-irrelevant communication. Clarifying these different impacts would facilitate the construction of a more optimal working environment.

Second, to avoid poor image focus, preclinical laparoscope navigation training seems desirable [23], thereby reducing sources of distraction brought by IT. Considering the results of the current study, basic skills training should not be restricted to tissue manipulation only. Rather, laparoscope navigation skills should be included in a comprehensive training program. Other sources of distraction brought by the introduction of IT in the operating room should be explored extensively considering the blooming of IT in the operating room.

Finally, individuals are unequal when facing distraction as a function of their propensity to be absorbed when using technology. Moreover, cognitively complex individuals are better able to deal with the complexity of the initial surgical task and the subsequently increased complexity under distracting conditions. These individual differences in cognitive style should be taken into consideration to provide an optimal working environment.

\section{Toward a comprehensive training program}

Experienced surgeons have gradually acquired coping strategies to deal with distractions while "training through reality." Using medical trainees in this study ensured the pure demonstration of the impact that social and technological distraction have on surgical performance in the absence of coping strategies. The introduction of myriad new IT in the operating room potentially adds distraction to the surgical process. Such distraction brought by IT results from inability to handle the IT properly (e.g., poor image focus), together with inability to filter and interpret the information IT brings to the surgical team, including unaddressed recipients (e.g., bleepers and alarms pertinent to anesthesiologists can be distracting to surgeons) and instrument failures. Although the latter is primarily an issue of technology maturity, usually referred to as equipment error, the other sources can be at least partially trained or managed.

Inversely, improper training in handling new IT essentially implies introducing more sources of distraction to the surgical process. Hence, training surgeons and surgical residents to manage (e.g., developing leadership skills) and to cope with plausible realistic distractions in a safe and controlled preclinical setting is paramount.
These efforts should complement training of both basic and IT-specific visual-spatial (e.g., coping with information presented by multiple screens) and psychomotor skills to provide a comprehensive and integrated training program.

Similar comprehensive training programs exist for anesthesia, and recent studies [24-26] suggest including cognitive training for surgeons. Accordingly, Helmreich [27] stresses the use of a deliberated training method tailored to conditions and considers human performance limiters such as fatigue and stress. Because the current study shows that individuals react differently to distracting sources, error training might even be tailored to individuals to avoid negative training. This will become even more important with the blooming of IT in the operating room.

Recommendations for further research

Further tests should involve experienced surgeons. Also, adding a third group of novices who trained in both basic psychomotor and coping skills would allow for objectification of the effect of the proposed comprehensive training program. In such studies, the authors opt to include realistic social and technological distractions to obtain relevant results. Distractions brought by IT comprise a rather unexplored but rapidly evolving source of potential realistic distraction in the current operating room.

Furthermore, in the minimal paradigm of this study, it was not possible to objectify the individual impact of the distracting sources, although the subjects indicated that they were irritated by both the social distraction and the nonoptimal laparoscope navigation. Testing a condition with proper laparoscope navigation and social distraction would be interesting for further research. A condition with nonoptimal laparoscope navigation in the absence of social distraction would, however, be unrealistic, because social distraction is virtually always present in the operating room $[7,22,28]$. Finally, a more comprehensive setting would possibly provide opportunities for physiologic measurement of acute stress levels during distracting conditions and complications.

\section{Conclusion}

The results of this study show a clear decline in task score and an increase in task errors and operating time when a laparoscopic task is performed under realistic distracting conditions. Additionally, individual differences in cognitive style (i.e., CA and NFC) appear to influence perceived irritation during performance. Working environment conditions in the operating room and preclinical training programs should cater accordingly by managing social and technological distracting sources and by providing 
comprehensive, integrated technical and nontechnical training programs. This will become even more important with the blooming of IT in the operating room.

Acknowledgments The authors thank the medical interns for participating in the experiment. Thanks also are due to Cees Schot and Sanne Botden at Catharina Hospital (Eindhoven, The Netherlands) for their generous technical assistance.

Disclosures J.R. Pluyter, S.N. Buzink, Drs. A.-F. Rutkowski, and J.J. Jakimowicz have no conflicts of interest or financial ties to disclose.

Open Access This article is distributed under the terms of the Creative Commons Attribution Noncommercial License which permits any noncommercial use, distribution, and reproduction in any medium, provided the original author(s) and source are credited.

\section{References}

1. Berguer R, Chen J, Smith WD (2003) A comparison of the physical effort required for laparoscopic and open surgical techniques. Arch Surg 138:967-970

2. Berguer R, Smith WD, Chung YH (2001) Performing laparoscopic surgery is significantly more stressful for the surgeon than open surgery. Surg Endosc 15:1204-1207

3. Cao CG, Taylor H (2004) Effects of new technology on the operating room team. In: Khalid HM, Helander MG, Yeo AW (eds). Proceedings of the 7th international conference on work with computing systems, special session on IT in Healthcare, Kuala Lumpur, Malaysia, June 29-July 2, Damai Sciences, pp 309-312

4. Healey AN, Primus CP, Koutantji M (2007) Quantifying distraction and interruption in urological surgery. Qual Saf Health Care 16:135-139

5. Healey AN, Sevdalis N, Vincent CA (2006) Measuring intraoperative interference from distraction and interruption observed in the operating theatre. Ergonomics 49:589-604

6. Primus CP, Healey AN, Undre S (2007) Distraction in the urology operating theatre. BJU Int 99:493-494

7. Sevdalis N, Healey AN, Vincent CA (2006) Distracting communications in the operating theatre. J Eval Clin Pract 13:390394

8. Buzink SN, Botden SMBI, Heemskerk J, Goossens RHM, de Ridder H, Jakimowicz JJ (2009) Camera navigation and tissue manipulation: are these laparoscopic skills related? Surg Endosc 23(4):750-757

9. Goodell KH, Cao CG, Schwaitzberg SD (2006) Effects of cognitive distraction on performance of laparoscopic surgical tasks. J Laparoendosc Adv Surg Techniques 16:94-98
10. Hsu K, Man FY, Gizicki R, Feldman L, Fried G (2008) Experienced surgeons can do more than one thing at a time: effect of distraction on performance of a simple laparoscopic and cognitive task by experienced and novice surgeons. Surg Endosc 22:196-201

11. Furnham A, Bradley A (1997) Music while you work: the differential distraction of background music on the cognitive test performance of introverts and extraverts. Appl Cognitive Psychol 11:445-455

12. Agarwal R, Karahanna E (2000) Time flies when you're having fun: cognitive absorption and beliefs about information technology usage. MIS Q 24:665-694

13. Cacioppo JT, Petty RE (1982) The need for cognition. J Pers Soc Psychol 42:116-131

14. Rutkowski A-F, Saunders CS (in review) Cognitive overload with IT: an emotional cognitive model towards IT adoption

15. Schijven MP, Jakimowicz J (2004) The learning curve on the Xitact LS 500 laparoscopy simulator: profiles of performance. Surg Endosc 18:121-127

16. Cohen S, Kessler RC, Gordon LU (1995) Measuring stress: a guide for health and social scientists. Oxford University Press, New York

17. Noble RE (2002) Diagnosis of stress. Metabolism 51:37-39

18. Zajonc RB (1965) Social facilitation. Science 149:269-274

19. Cohen S, Kamarck T, Mermelstein R (1983) A global measure of perceived stress. J Health Soc Behav 24:385-396

20. Monroe SM, Kelley JM (1995) Measurement of stress appraisal. In: Cohen S, Kessler RC, Underwood G (eds) Measuring stress: a guide for health and social scientists. Oxford University Press, New York, pp 122-147

21. Moorthy K, Munz Y, Dosis A, Bann S, Darzi A (2003) The effect of stress-inducing conditions on the performance of a laparoscopic task. Surg Endosc 17:1481-1484

22. Moorthy K, Munz Y, Undre S, Darzi A (2004) Objective evaluation of the effect of noise on the performance of a complex laparoscopic task. Surgery 136:25-30

23. Dunkin B, Adrales GL, Apelgren K, Mellinger JD (2007) Surgical simulation: a current review. Surg Endosc 21:357-366

24. Moorthy K, Munz Y, Forrest D, Pandey V, Undre S, Vincent C, Darzi A (2006) Surgical crisis management skills training and assessment: a simulation[corrected]-based approach to enhancing operating room performance. Annu Surg 244:139-147

25. Yule S, Flin R, Paterson-Brown S, Maran N (2006) Nontechnical skills for surgeons in the operating room: a review of the literature. Surgery 139:140-149

26. Kahol K, Vankipuram M, Smith ML (2009) Cognitive simulators for medical education and training. J Biomed Inform 42:593-604

27. Helmreich RL (2000) On error management: lessons from aviation. BMJ 320:781-785

28. Mahawar KK (2003) Too much talk in theatre. Lancet $361: 1570$ 